\title{
Black hole acoustics in the minimal geometric deformation of a de Laval nozzle
}

\author{
Roldão da Rocha ${ }^{\mathrm{a}}$ \\ Centro de Matemática, Computação e Cognição, Universidade Federal do ABC-UFABC, Santo André 09210-580, Brazil
}

Received: 5 March 2017 / Accepted: 15 May 2017 / Published online: 29 May 2017

(C) The Author(s) 2017. This article is an open access publication

\begin{abstract}
The correspondence between sound waves, in a de Laval propelling nozzle, and quasinormal modes emitted by brane-world black holes deformed by a 5D bulk Weyl fluid are here explored and scrutinized. The analysis of sound waves patterns in a de Laval nozzle in the laboratory, reciprocally, is here shown to provide relevant data about the $5 \mathrm{D}$ bulk Weyl fluid and its on-brane projection, comprised by the minimal geometrically deformed compact stellar distribution on the brane. Acoustic perturbations of the gas fluid flow in the de Laval nozzle are proved to coincide with the quasinormal modes of black holes solutions deformed by the $5 \mathrm{D}$ Weyl fluid, in the geometric deformation procedure. Hence, in a phenomenological Eötvös-Friedmann fluid brane-world model, the realistic shape of a de Laval nozzle is derived and its consequences studied.
\end{abstract}

\section{Introduction}

General relativity (GR) is a successful theory, widely tested by experiments and observations, however a limited one as regards some recent questions, like the nature of dark energy/dark matter. GR can be recovered from models involving higher dimensions as a very restricted case [1,2]. In brane-world models, the brane self-energy density is manifest as the brane tension $(\sigma)$, which is assumed to be infinite in the GR limit. Nevertheless, a finite value for the brane tension, in codimension one models, is an ubiquitous feature of the brane that also comprises the warped five-dimensional (5D) geometry, besides the brane self-gravity.

Although an enormous brane tension value recovers GR at low energy regimes, phenomenological evidence indicates a variable brane tension [3-6]. At an immensely hot universe, the tension of the brane had attained a nugatory value. Afterwards, the brane tension increased and the universe cooled down [3-5]. Fluid membranes play a central role in model-

a e-mail: roldao.rocha@ufabc.edu.br ing this scenario, wherein a temperature-dependent tension is ruled by the Eötvös principle [6], which governs a Friedmann brane with a scale factor that drives the expansion of the universe [7].

4D gravity can be effectively formulated on a brane-world by two complementary methods. The first one comprises the Shiromizu-Maeda-Sasaki implementation of the GaussCodazzi on-brane projection routine [8]. Nevertheless, this method does not represent a consistent system of equations, since the 5D bulk Weyl tensor cannot be determined from data on the brane. In fact, there is no action whose projected Euler-Lagrange equations onto the brane can be derived [2]. A complementary technique does involve an action that, at low energy regimes, derives the effective 4D theory described by the respective Euler-Lagrange equations [9]. Hence, these two complementary procedures can be employed in the construction of an effective 4D theory [5].

Among successful efforts to formulate theories beyond GR, the procedure consisting in accomplishing a minimal geometric deformation of the Schwarzschild solution in a brane-world was derived [10-12]. It comprises exact solutions of the Einstein equations for the 4D effective theory on the brane [10-12]. The minimal geometric deformation procedure incorporates high-energy improvements to GR, when the (brane) vacuum state is percolated by a 5D Weyl fluid in the bulk $[10,13,14]$. These analytical solutions - of the 4D brane effective Einstein's field equations - encode compact distributions supporting stellar systems that can even exhibit solid crusts $[13,14]$, driven by the 5D bulk Weyl fluid, and also peculiar generalizations [15]. The deformation itself comprises the brane tension as the managing parameter of high-energy regimes. This setup has GR corresponding to an ideally rigid brane $(\sigma \rightarrow \infty)$, at low energies. A more refined setup can be implemented by considering a variable tension fluid brane $[3,16]$. This approach has been comprehensively and successfully constrained by experimental and observational bounds, provided by the perihelion precession of Mer- 
cury, the deflection of light by the Sun, the gravitational redshift and the radar echo delay, recently obtained in Ref. [17]. Besides, observational lensing effects by minimal geometrically deformed black holes have a typical signature that may soon be probed by the European Space Agency satellite mission [18]. Despite this comprehensive list of experimentally and observationally possible signatures, regarding the brane-world black hole that is geometrically deformed by a 5D Weyl fluid, any study of the quasinormal modes produced by this kind of black holes is still lacking.

On the other hand, acoustic perturbations of a gas flow, in the so-called de Laval nozzle, have been shown to correspond to the general form of perturbations of Schwarzschild black holes [19-21]. It introduces the feasibility to produce and observe their quasinormal resonances in the laboratory. The de Laval nozzle is an example among propelling nozzles, which are widely studied devices that turn a fluid (gas) turbine into a jet engine. A de Laval nozzle is constituted by an hour-glass-shaped tube, strained in the middle, utilized to accelerate a hot pressurized gas to a high supersonic speed into the thrust direction. The fluid thermal energy is commuted into kinetic energy, and the fluid velocity increases. The energy to accelerate the gas stream induces the gas to adiabatically expand with high efficiency to a final - transonic, supersonic, or even hypersonic speed - propelling jet. The de Laval nozzle is constructed upon the theory of quasi1D flows, where a fluid moves at the magnitude of the speed of sound. In this regime, the changes in the fluid density turn significant and compress the flow. The de Laval nozzle is based upon the Venturi effect.

Fluid flows have been studied, in this context, in a de Laval nozzle, aiming to observe acoustic black holes in the Schwarzschild setup [19-21]. The acoustic black hole surface gravity was experimentally derived in the laboratory, in Ref. [22]. As argued in Refs. [20,21], sonic regions in a fluid can induce a surface for the sound waves, known as the acoustic horizon, that emulates a black hole event horizon. Perturbations of sound waves have been shown to be ana$\log$ to the quasinormal modes, corresponding to black holes gravitational excitations [23,24], being, moreover, successfully explored in different contexts [25-27].

Our point here is to derive and analyze the correction to a de Laval nozzle trend, using also its analogy to a braneworld black hole in the minimal geometric deformation setup, regarding a variable brane tension. Besides, another goal here is to study the analogy between waves in a de Laval nozzle in the laboratory and quasinormal modes of minimal geometrically deformed brane-world black holes. Hence, to scrutinize sonic waves in a de Laval nozzle can circumvent the indeterminacy of the 5D Weyl tensor on the bulk $[2,28]$, which encrypts the bulk geometry. Since the minimal geometric deformation is generated by a 5D Weyl fluid on the bulk, experiments regarding a de Laval nozzle in the labo- ratory may reciprocally provide relevant data about the $5 \mathrm{D}$ bulk Weyl fluid.

This paper is organized as follows: Sect. 2 is devoted to a brief review, regarding minimal geometrically deformed compact systems, further refined by phenomenological Eötvös-Friedmann fluid branes. In Sect. 3, a gas flow is perturbed in a de Laval nozzle, whose wave equation is analog to the wave equation regarding spin- $s$ perturbations of minimal geometrically deformed brane-world black holes. Hence, the current bound for the variable brane tension provides corrections to the expression for the trend of de Laval nozzles. Moreover, we here propose to study quasinormal modes from these black holes in the laboratory, when the wave equation in a de Laval nozzle equals the wave equation of spin-s perturbations of brane-world black holes undergoing a minimal geometric deformation. Section 4 is dedicated to a discussion of our results, to summarizing the conclusions and to providing relevant perspectives.

\section{The minimal geometric deformation setup and fluid branes}

Acoustic analogs of brane-world black holes have been reported [29], in the context of setups due to both Randall and Sundrum [30] and to Dvali et al. [31]. However, no approach has regarded realistic data on the brane yet, comprising the variable brane tension paradigm on fluid branes. Employing the minimal geometric deformation technique incorporates high-energy refinements to general relativity, by permeating the brane vacuum outer of a compact distribution with a Weyl fluid in the 5D bulk [10,13,14]. Brane-world models encompassing a variable brane tension are best implemented by Eötvös-Friedmann fluid branes, where the brane temperature drives the brane tension, according to the Eötvös' rule across the universe expansion $[3,6]$.

The most stringent brane tension bound $\sigma \gtrsim 3.2 \times$ $10^{-6} \mathrm{GeV}^{4}$ has been provided in the context of the minimal geometric deformation of black holes, formed as BoseEinstein condensates of gravitons that weakly interact among themselves [32]. The associated entropic information content can also predict the Chandrasekhar critical density of compact objects in this paradigm $[32,33]$.

The effective 4D Einstein equations can be derived by the Gauss-Codazzi on-brane projection method, from the 5D bulk equations $[2,8]$. In natural units, the 4D Einstein effective equations were obtained in Ref. [8] (Greek indices run in the set of Minkowski space-time indices):

$G_{\mu \nu}+\Lambda g_{\mu \nu}-\mathrm{T}_{\mu \nu}=0$,

where $G_{\mu \nu}$ denotes the Einstein tensor and the cosmological parameter on the brane is denoted by $\Lambda$. The effective stress- 
energy tensor $\mathrm{T}_{\mu \nu}=T_{\mu \nu}+\sigma^{-1} S_{\mu \nu}+\mathcal{E}_{\mu \nu}+P_{\mu \nu}+L_{\mu \nu}$ encrypts the matter stress-energy tensor on the brane $\left(T_{\mu \nu}\right)$, the 5D bulk Weyl tensor electric projection on the brane $\left(\mathcal{E}_{\mu \nu}\right)$ - that comprise data (constituting a Weyl fluid) about the gravitational field out of the brane - and $S_{\mu \nu}$ is the traceless irreducible component, proportional to the brane extrinsic curvature [8] - regards 5D effects onto the brane; the tensor components $L_{\mu \nu}$ encrypt the asymmetric embedding of the brane, into the bulk, and $P_{\mu \nu}$ stands for the pullback onto the brane of the stress-energy tensor, designating eventual 5D non-standard model fields, comprising radiation of quantum origin, dilatonic, and even moduli fields $[2,7,8]$. Deviations from the usual Einstein standard equations in GR can also be generated by excitations of 5D gravitons, whose effects are encompassed in the $P_{\mu \nu}$ tensor. Eventually, some of the terms constituting $\mathrm{T}_{\mu \nu}$ can equal zero.

Exact solutions of the 4D effective Einstein equations are rare, due to the intricacy of the system of equations and to initial data out of the brane as well. Compact distributions, modeling stellar structures, are spherically symmetric, static solutions of Eq. (1), of type

$\mathrm{d} s^{2}=-A(r) \mathrm{d} t^{2}+(B(r))^{-1} \mathrm{~d} r^{2}+r^{2} \mathrm{~d} \Omega^{2}$,

for $\mathrm{d} \Omega^{2}$ representing the 2 -sphere surface element. The deformation on the radial component in Eq. (2) is caused by the bulk constituents, encrypting not only anisotropic effects originating from the bulk gravity but also the effects of a 5D Weyl fluid in the bulk, whose brand permeates the brane vacuum.

The minimal geometric deformation is implemented by fixing the temporal component in (2) and deforming the outer radial component $[12,14]$,

$B(r)=1-\frac{2 M}{r}+\varsigma \mathrm{e}^{\Theta(r)}$,

where

$\Theta(r)=\int_{R}^{r} \frac{f_{1}(A(a))}{f_{2}(A(a))} \mathrm{d} a$,

for [12]

$f_{1}(A)=\frac{\ln (A)^{\prime}}{r^{2}}\left(\ln (A)^{\prime}+\frac{2}{r}\right)+\frac{A A^{\prime \prime}}{A^{\prime 2}}-1$,

$f_{2}(A)=\left(\frac{2}{r}+\frac{1}{2} \ln (A)^{\prime}\right)^{-1}$.

The prime denotes the derivative with respect to the radial coordinate, and $R$ denotes the compact distribution effective radius [10]. The $\varsigma$ parameter in (3) regards the Weyl fluid in the bulk and its induced deformation of the brane 4D vacuum [17]. The region inner to the stellar distribution is regular at the origin. The inner and outer region to a star have a shared boundary constituted of a solid incrustation. The (variable) brane tension and the stellar effective radius are parameters that determine the star crust width $[13,14]$. The outer region $r>R[10]$ then promotes the deformed metric [12]

$A(r)=1-\frac{2 M}{r}$,

$B(r)=\left[1+\frac{\varsigma^{l}}{r\left(1-\frac{3 M}{2 r}\right)}\right]\left(1-\frac{2 M}{r}\right)$,

where [12] $\mathfrak{l} \equiv\left(1-\frac{2 M}{\mathrm{R}}\right)^{-1}\left(1-\frac{3 M}{2 R}\right) R$. References [10, 16] show that the metric radial component (6a) can be split as follows - in this section the subindex " 0 " refers to the GR limit $\sigma \rightarrow \infty$ (or, equivalently, $\varsigma=0$ ):

$B(r)=B_{0}(r)+B_{\varsigma}(r)$,

where

$B_{0}(r)=\lim _{\varsigma \rightarrow 0} B(r)=1-\frac{2 M}{r}$,

$B_{\zeta}(r)=-\frac{\left(1-\frac{2 M_{0}}{r}\right) \frac{1}{r}}{1-\frac{3 M_{0}}{2 r}}\left(\mathfrak{l}_{M_{0}}\right) \varsigma$,

where $B_{\zeta}(r)$ evinces a high-energy correction to the Schwarzschild solution to order $\mathcal{O}\left(1 / \sigma^{2}\right)$, for $M=M_{0}+$ $\mathcal{O}(1 / \sigma)$.

The parameter $\varsigma$ is proportional to the stellar distribution compactness and drives the geometric deformation of the Schwarzschild solution, having an explicit expression in terms of the brane tension $[10,12]$ :

$$
\begin{aligned}
\varsigma & \approx \frac{\alpha \tau(\mathrm{R})}{98 \pi^{2} \sigma} \frac{\left(\left.\mathfrak{l}\right|_{M_{0}}\right)}{R}\left[63 \alpha+390 \alpha^{2}-\frac{9167}{7} \alpha^{3}+\frac{135,952}{63} \alpha^{4}\right] \\
& \propto \frac{\sigma^{-1}}{\mathrm{R}^{2}}\left(\left.\mathfrak{l}\right|_{M_{0}}\right) \equiv-d_{0} \frac{\sigma^{-1}}{R}
\end{aligned}
$$

for $\alpha \equiv d_{0} R^{2}$ and

$\tau(r) \equiv\left(1+\alpha\left(\frac{r}{\mathrm{R}}\right)^{2}\right)^{-3}\left(1+3 \alpha\left(\frac{r}{\mathrm{R}}\right)^{2}\right)^{-1}$

Typically $d_{0} \approx \frac{0.27492}{R^{2}}$. The GR $\sigma \rightarrow \infty$ limit yields $\varsigma=0$ in Eqs. (6a) and (6b), leading to the standard Schwarzschild metric solution of Einstein's equations.

The current experimental and observational data was shown to enforce the strongest bound $|\varsigma| \lesssim 6.1 \times 10^{-11}$ (obtained by the perihelion precession classical test of GR) and the weakest bound $|\zeta| \lesssim 8.2 \times 10^{-5}$ (derived from the radar echo delay classical test of GR) on the adimensional deformation parameter, in Ref. [17]. Besides, the most recent brane tension bound $\sigma \gtrsim 3.2 \times 10^{-6} \mathrm{GeV}^{4}$ has been obtained by the informational entropy of the minimal geometrically deformed Bose-Einstein condensate of gravitons [32]. Equation (9) implies a negative value for the parameter 5 . Hence, the gravitational field strength is mitigated by the finite value of the brane tension and by the 5D encompassing scenario 
[17], which attains a maximum at the stellar surface $r=R$. Denoting the stellar distribution density by $\rho_{\text {star }}$, the bound $\sigma \gtrsim 3.2 \times 10^{-6} \mathrm{GeV}^{4}$ complies with the condition $\frac{\rho_{\text {star }}}{\sigma} \ll 1$ $[2,3]$.

Eötvös-Friedmann fluid branes are known to have a variable brane tension proportional to the temperature of the universe, $T$. The Eötvös law asserts that $\sigma \approx T-\tau$, [3,34], for $\tau$ a crucial constant parameter that drives $\sigma$ into positive values, subsequently to the Big Bang $[3,6]$. The scale factor constant value $a_{0}$ fixes the beginning of the universe at a $\tau$ temperature [3,34]. Reference [3] computed the temperature dependence upon the scale factor as $T(t) \approx \frac{1}{a(t)}$ [3], yielding a variable brane tension that is time dependent $[3,4]$,

$\frac{\sigma(t)}{\sigma_{0}}=\frac{\kappa^{2}}{\kappa_{0}^{2}}=1-\frac{a_{0}}{a(t)}$,

where $\kappa^{2}$ denotes the 4D coupling "constant" and $\kappa_{0}^{2}=$ $(8 \pi G)^{-1}$ is the late-time coupling constant, $G$ denoting the Newton's constant.

At early times, until the radiation density equated the brane matter density, the brane tension could be taken as having a low value. However, the brane tension and the 4D coupling parameter likewise magnified as the universe expanded. The time-dependent brane tension expression yields $\Lambda_{4 \mathrm{D}}=$ $\Lambda_{0}-\frac{a_{0}}{a(t)}\left(1-\frac{a_{0}}{2 a(t)}\right) \kappa_{0}^{2} \sigma_{0}[3,4]$. Black string and black brane solutions in a variable brane-world context were studied in Refs. [16,35,36].

\section{The minimal geometric deformation of a de Laval nozzle}

A de Laval nozzle is intrinsically based on the Venturi effect. When the flow of a (gas) fluid passes through a constricted part of a tube with variable cross section $A(x)$, it generates a reduction in the fluid pressure, whereas the fluid velocity increases. Modeling de Laval nozzles considers quasi-1D flows, which are isentropic, adiabatic, and frictionless ones; these shall be considered here. The fluid studied can consist of an ideal gas and expressed by the equation of state $p=\rho R T$, where $p$ denotes the fluid pressure, $T$ is the absolute temperature, and $R$ is the universal gas constant. An ideal gas is well known to have a constant heat capacity, at constant pressure and constant volume - respectively denoted by $C_{\mathrm{p}}$ and $C_{\mathrm{V}}$. Hence $R=C_{\mathrm{p}}-C_{\mathrm{V}}$ and the specific heat ratio reads $\gamma=C_{\mathrm{p}} / C_{\mathrm{V}}$. For instance, the heat capacity ratio for helium is $\gamma=1.66$, whereas nitrogen has $\gamma=1.4$. Here diatomic gas molecules shall be regarded.

An isentropic gas flow, from an initial to a final state, has the property [37]

$p=\rho^{\gamma}=T^{\frac{\gamma}{\gamma-1}}$, where these quantities shall be normalized by the initial state. Prominent properties of isentropic flows comprise the uniform expansion of the gas, composing then a shock-free, continuous, flow. A relevant parameter of a compressible flow is the Mach number, $\mathfrak{M}(x)=\frac{v(x)}{c_{s}(x)}$, where $c_{s}^{2}=\frac{\mathrm{d} p}{\mathrm{~d} \rho}=\gamma R T$ is the local speed of sound and $x$ denotes the transversal nozzle coordinate, namely, the coordinate along the de Laval nozzle, and $v$ is the local flow velocity. The Mach number is employed to categorize the distinct regimes of flow. ${ }^{1}$ Besides, the mass flow rate $\frac{\mathrm{d} m}{\mathrm{~d} t}$ is the flux per unit throat area $\rho A v$, meaning the mass of the gas that passes through a cross section of the tube per unit time, also known as the fluid discharge [37]. A quasi-1D fluid flow is ruled by the Euler-Lagrange equations and the continuity relation in fluid mechanics, given by [37]

$$
\begin{aligned}
& \frac{\partial}{\partial t}(\rho A)+\frac{\partial}{\partial x}(\rho A v)=0, \\
& \frac{\partial}{\partial t}(\rho A v)+\frac{\partial}{\partial x}\left[\left(\rho v^{2}+p\right) A\right]=0 \\
& \frac{\partial}{\partial t}\left(\frac{\rho v^{2}}{2}-\frac{p}{1-\gamma} A\right)+\frac{\partial}{\partial x}\left[\left(\frac{\rho v^{2}}{2}-\frac{\gamma}{1-\gamma}\right) A v\right]=0 .
\end{aligned}
$$

Instead of Eq. (13b), one can use the Euler equation

$\rho\left(\frac{\partial v}{\partial t}+v \frac{\partial v}{\partial x}\right)+\frac{\partial p}{\partial x}=0$,

associated with the Bernoulli equation

$\frac{1}{2}\left(\frac{\partial \Phi}{\partial x}\right)^{2}+\int \rho^{-1} \mathrm{~d} p=-\frac{\partial \Phi}{\partial t}$,

where the last term in Eq. (15) represents the heat function of a barotropic fluid, identified with the enthalpy, and $\Phi=\int v \mathrm{~d} x$ denotes the velocity potential. From Eq. (15), the linearized equation for sound waves can then be obtained, considering perturbations $\phi$ and $\delta \rho$, respectively, around $\Phi$ and $\rho[19,20]$.

A quasi-1D fluid flow in a de Laval nozzle has a stagnation state, which is a state attained by the fluid if it is conveyed to rest into an isentropic state and without work. The stagnation speed of sound is denoted by $c_{s 0}$. Now, the acoustic analog of the tortoise coordinate, $x^{\star}$, is defined by

$x^{\star}=c_{s 0} \int\left[c_{s}(x)\left(1-\mathfrak{M}(x)^{2}\right)\right]^{-1} \mathrm{~d} x$.

Perturbing the system of equations (13a)-(13c) in a nozzle yields [19]

$\left[\frac{\mathrm{d}^{2}}{\mathrm{~d} x_{\star}^{2}}+\frac{\omega^{2}}{c_{s 0}^{2}}-V\left(x_{\star}\right)\right] \phi\left(\omega, x_{\star}\right)=0$,

\footnotetext{
1 Those regimes include hypersonic, supersonic, transonic, sonic, and subsonic flows.
} 
where the associated potential, representing the sound waves curvature scattering on the acoustic black hole, reads

$V\left(x_{\star}\right)=\frac{1}{2 g^{2}}\left(g \frac{\mathrm{d}^{2} g}{\mathrm{~d} x^{\star 2}}-\left(\frac{\mathrm{d} g}{\sqrt{2} \mathrm{~d} x_{\star}}\right)^{2}\right)$,

for $[19,20]$

$g(x) \equiv \frac{\rho(x) A(x)}{c_{S}(x)} \underset{\sim}{\sim} \frac{A(x)}{2 \rho^{(\gamma-3) / 2}}$,

$\phi\left(\omega, x_{\star}\right)=\int \sqrt{g\left(x_{\star}\right)} \phi\left(t, x_{\star}\right) \mathrm{e}^{i \omega\left[t-f\left(x_{\star}\right)\right]} \mathrm{d} t$,

where $f(x)$ in Eq. (20) is a function defined by $\frac{\mathrm{d} f(x)}{\mathrm{d} x}=$ $\frac{|v|}{c_{s}^{2}-v^{2}}$.

A de Laval nozzle trend is constructed upon the nozzle throat cross-sectional area. Dimensionless quantities for $\rho(x)$ and $A(x)$ are obtained by measuring them in units of the stagnation gas density $\rho_{0}$ and of the throat nozzle crosssectional area, respectively. Moreover [20,21],

$A \stackrel{\sim}{\sim}\left(1-\rho^{(\gamma-1)}\right)^{1 / 2} \rho$,

which by Eq. (19) yields $g=\frac{\rho^{1-\gamma}}{2\left(\rho^{1-\gamma}-1\right)^{1 / 2}}$, following which we have

$\rho^{1-\gamma}=2 g^{2}-2 g \sqrt{g^{2}-1}=1+\frac{\gamma-1}{2} \mathfrak{M}^{2} \geq 1$,

yielding

$\mathfrak{M}^{2}=\frac{2}{\gamma-1}\left(2 g^{2}-2 g \sqrt{g^{2}-1}-1\right)$.

The Mach number equals the unit at the horizon, wherein thus $\mathrm{g}$ has to be finite,

$g_{\text {horizon }}=\frac{1+\gamma}{2 \sqrt{2 \gamma-2}}=\frac{3 \sqrt{5}}{5} \geq 1$.

Replacing Eq. (22) into Eq. (19) implies the cross-sectional nozzle area expressed in terms of $g$ [19],

$$
\begin{aligned}
A & =2^{\frac{1+\gamma}{2 \gamma-2}} \mathrm{~g}^{\frac{4-\gamma}{2 \gamma-2}}\left(g-\sqrt{g^{2}-1}\right)^{\frac{2-\gamma}{\gamma-1}}, \\
& =\frac{1}{\mathfrak{M}^{2}}\left[\left(1+\frac{\gamma-1}{2} \mathfrak{M}^{2}\right) \frac{2}{\gamma+1}\right]^{\frac{1+\gamma}{\gamma-1}} .
\end{aligned}
$$

On the other hand, the analogy between fluid flows in a de Laval nozzle and the brane-world black hole - undergoing a minimal geometric deformation - can be implemented. In fact, scalar field perturbations in the minimally geometric deformed brane-world black hole background are known to yield the wave-like equation [25]

$\left(\frac{\mathrm{d}^{2}}{\mathrm{~d} r_{*}^{2}}+\omega^{2}-V\left(r_{*}\right)\right) \Psi\left(r_{*}\right)=0$, where $\mathrm{d} r_{*}=\frac{\mathrm{d} r}{B(r)}$ and the effective potential for the quasinormal ringing of the brane-world black hole under the minimal geometric deformation reads

$$
\begin{aligned}
V(r)= & \left(1+\frac{\varsigma^{l}}{r\left(1-\frac{3 M}{2 r}\right)}\right)\left(1-\frac{2 M}{r}\right)\left\{\frac{\ell(\ell+1)}{r^{2}}\right. \\
& +\left(1-s^{2}\right)\left[2 M\left(1+\frac{\varsigma^{l}}{r\left(1-\frac{3 M}{2 r}\right)}\right)\right. \\
& \left.\left.-\frac{\varsigma^{l}}{r^{2}\left(1-\frac{3 M}{2 r}\right)^{2}}\left(1-\frac{2 M}{r}\right)\right] \frac{1}{r^{3}}\right\} .
\end{aligned}
$$

Equation (27) is analog to Eq. (17). In fact, to find a scalar function $g$ that produces the same potential, the tortoise coordinate of the black hole solution is identified with the de Laval nozzle, $\mathrm{d} r_{*}=\mathrm{d} x_{\star}$, yielding

$$
\begin{aligned}
\mathrm{d} x_{\star}^{2} & =\frac{\rho^{1-\gamma}}{\left(1-\mathfrak{M}^{2}\right)^{2}} \mathrm{~d} x^{2} \\
& =\frac{2 g^{2}-2 g \sqrt{\mathrm{g}^{2}-1}-1}{\left[1-\frac{2}{\gamma-1}\left(2 \mathrm{~g}^{2}-2 g \sqrt{\mathrm{g}^{2}-1}-1\right)-1\right]^{2}} \mathrm{~d} x^{2} .
\end{aligned}
$$

The differential equation for $g(r)$ then reads

$\left[B(r) g^{\prime}(r)\right]^{\prime}-B(r) B^{\prime}(r) \mathrm{g}^{\prime}(r)-\frac{\left(B(r) g^{\prime}(r)\right)^{2}}{2 \mathrm{~g}(r)}=V(r) g(r)$.

One can elect an unit event horizon radius, so that the nozzle coordinate to be written with respect to the event horizon.

In the limit $\varsigma \rightarrow 0$, Eq. (7) is reduced to Eq. (30) in Ref. [20], which has the solution in Eq. (31) in that reference. Substituting Eq. (7) into (30) yields an intricate equation that cannot be analytically solved. However, by splitting the solution of (30) into a sum of a purely GR component $\left(\mathrm{g}_{0}(r) \equiv \lim _{\sigma \rightarrow \infty} g(r)\right)$ and a component that is induced by the 5D Weyl fluid,

$g(r)=g_{0}(r)+g_{\zeta}(r)$,

we can substitute the solution of (30) for $\varsigma=0$,

$\mathrm{g}_{0}(r)=\frac{1+\gamma}{2 \sqrt{2 \gamma-2}} \sum_{j=s}^{\ell}\left(\frac{(\ell+j) !}{(j-s) !(s+j) !(\ell-j) !} r^{j+1}\right)^{2}$,

obtained in Ref. [20], to find the $\mathrm{g}_{5}(r)$ function, iteratively solving Eq. (30). The solution of Eq. (30) has two integration constants, determined by Eq. (24). Equations (29) and (6b) then provide the nozzle coordinate $x$ with respect to $r$, 


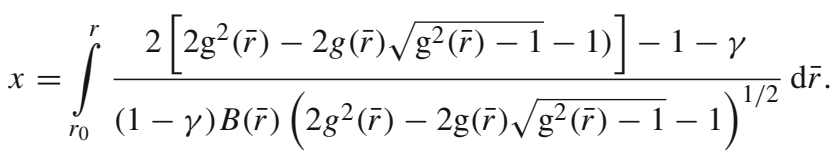

The integral lower limit can be made consistent with the fact that the coordinate $x$ is null at the sonic point, by imposing $r_{0}=1$.

Hence, the de Laval nozzle cross section $A(x)$ can finally be derived, modeling the nozzle shape. In fact, Eq. (32) can be put into Eq. (30), whose numerical solutions for $\mathrm{g}(r)$ yield the corrections (due to the 5D Weyl fluid) $\mathrm{g}_{5}(r)$, in Eq. (31). Subsequently, we rewrite the numerical solution $g(r)$ in Eq. (31) with respect to the transversal nozzle coordinate $x$ in Eq. (32), substituting it into the expression for the nozzle cross section $A(x)$ in Eq. (26).

In what follows, the solid gray areas in Figs. 1, 2 and 3 indicate the cross section $A(x)$ of the de Laval nozzle and its shape, in the $\sigma \rightarrow \infty$ GR limit (gray area limited by the continuous gray line) and its minimal geometric deformation due to a 5D bulk Weyl fluid (gray area limited by the dotted gray line). The black strips, respectively, represent the effective potential for those two cases. The brane tension bound adopted, $\sigma \approx 3.2 \times 10^{-6} \mathrm{GeV}^{4}$, was derived in Ref. [32] through the numerical computation of the minimal geometric deformation case, as well as the weakest experimental

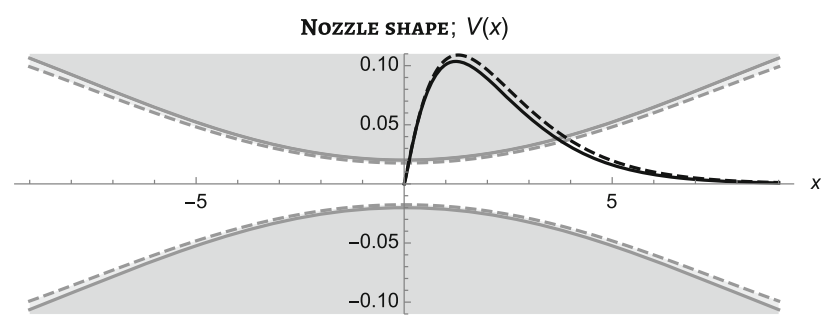

Fig. 1 The nozzle profile for $s=\ell=0$. The gray filled area, limited by the continuous (dashed) line denotes the de Laval nozzle in the Schwarzschild, GR $\sigma \rightarrow \infty$, limit (in the minimal geometric deformation of the de Laval nozzle). The black lines represent $V(x)$ for the GR limit (continuous line) and its minimal geometric deformation (dashed line)

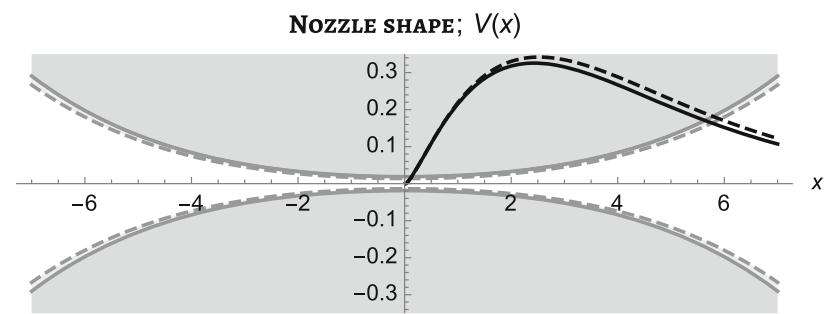

Fig. 2 The nozzle profile for $s=\ell=1$. The gray filled area, limited by the continuous (dashed) line denotes the de Laval nozzle in the Schwarzschild, GR $\sigma \rightarrow \infty$, limit (in the minimal geometric deformation of the de Laval nozzle). The black lines represent $V(x)$ for the GR limit (continuous line) and its minimal geometric deformation (dashed line)

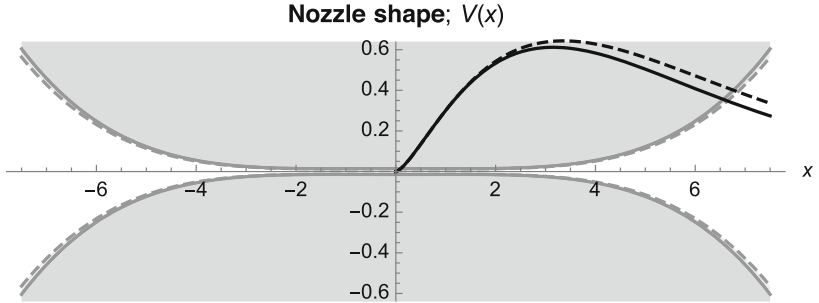

Fig. 3 The nozzle profile for $s=\ell=2$. The gray filled area, limited by the continuous (dashed) line denotes the de Laval nozzle in the Schwarzschild, GR $\sigma \rightarrow \infty$, limit (in the minimal geometric deformation of the de Laval nozzle). The black lines represent $V(x)$ for the GR limit (continuous line) and its minimal geometric deformation (dashed line)

bound $|\varsigma| \lesssim 8.2 \times 10^{-5}$ on the minimal geometric deformation parameter [17].

The minimal geometric deformation constricts the nozzle throat cross-sectional area. In addition, by comparing the Schwarzschild versus minimal geometric deformation, the nozzle corrections due to the influence of a 5D bulk Weyl fluid that permeates the vacuum on the brane are notorious. Hence, the quasinormal modes of black holes solutions deformed by the 5D bulk Weyl fluid can be probed by their analogy with acoustic perturbations of a diatomic gas fluid flow in a de Laval nozzle, using the geometric deformation technique. Reciprocally, the signature and the lacking data about the Weyl tensor in the bulk can be probed in the laboratory, by analyzing sonic waves throughout a minimal geometrically deformed de Laval nozzle. The next section is devoted to further exploring and analyzing the consequences of our results.

\section{Concluding remarks and outlook}

The minimal geometric deformation of a de Laval nozzle can have double-handed applications. The de Laval nozzle associated with black hole analogs produced in the laboratory can present their trend slightly modified by 5D bulk Weyl fluid effects. On the other hand, 5D effects can also be probed in the laboratory, due to the analogy presented here.

Using a phenomenological Eötvös-Friedmann fluid brane setup, describing an inflationary brane-world universe, the perturbation of a fluid flow in a de Laval nozzle was considered, providing a wave equation that is similar to the wave equation regarding perturbations of minimal geometrically deformed brane-world black holes. The precise bounds for the variable brane tension value provided corrections to the shape of de Laval nozzles in this context. Figures 1, 2 and 3 plot the de Laval nozzle profile and also the analysis of the nozzle deformation with respect to the Schwarzschild solution. Such a deformation is generated by a 5D Weyl fluid permeating a compact distribution described by the Schwarzschild metric solution of the 4D brane effective Einstein equations. 
Moreover, quasinormal modes of brane-world black holes undergoing a minimal geometric deformation can then be produced and observed in the laboratory, by analyzing the sonic waves throughout the associated deformed Laval nozzle. Hence, the solution for the inverse technique, consisting of the correspondence between the shape of the de Laval nozzle and the general trend of perturbations in brane-world black holes deformed by a minimal geometric deformation of Schwarzschild black holes, has been implemented here. The corrections to the Schwarzschild solution on the brane, permeated by a 5D bulk Weyl fluid, affect how the pressure is dispersed across the deformed de Laval nozzle. The finite brane tension then specifies a protocol for the analysis telling us whether the de Laval nozzle highest thrust can be achieved and where we search for the best flow properties that are being attained, for the derived de Laval nozzle shape.

Using the sonic analog to black holes [38], the thermal spectrum of sound waves was given from the sonic horizon in transonic fluid flows, also in the context of analog gravity [39-41]. These approaches can be further explored, using the methods here introduced, together with more fluid analogy phenomena regarding black holes in the laboratory [42,43]. Still, further types of black holes can be studied [27,44]. Finally, the extended MGD approach [45] can also be used to derive further corrections to the de Laval nozzle profile.

Acknowledgements RdR is grateful to CNPq (Grant No. 303293/ 2015-2), to FAPESP (Grant No. 2015/10270-0), for partial financial support, and to Dr. A. Zhidenko for valuable discussions.

Open Access This article is distributed under the terms of the Creative Commons Attribution 4.0 International License (http://creativecomm ons.org/licenses/by/4.0/), which permits unrestricted use, distribution, and reproduction in any medium, provided you give appropriate credit to the original author(s) and the source, provide a link to the Creative Commons license, and indicate if changes were made.

Funded by SCOAP ${ }^{3}$.

\section{References}

1. I. Antoniadis, N. Arkani-Hamed, S. Dimopoulos, G.R. Dvali, Phys. Lett. B 436, 257 (1998). arXiv:hep-ph/9804398

2. R. Maartens, K. Koyama, Liv. Rev. Rel. 13, 5 (2010). arXiv: 1004.3962

3. L.A. Gergely, Phys. Rev. D 79, 086007 (2009). arXiv:0806.4006

4. L.A. Gergely, Phys. Rev. D 78, 084006 (2008). arXiv:0806.3857

5. S. Kanno, J. Soda, Gen. Rel. Grav. 36, 689-712 (2004). arXiv:hep-th/0303203

6. M.C.B. Abdalla, J.M. Hoff da Silva, R. da Rocha, Phys. Rev. D 80, 046003 (2009). arXiv:0907.1321

7. L.A. Gergely, Phys. Rev. D 68, 124011 (2003). arXiv:gr-qc/0308072

8. T. Shiromizu, K.I. Maeda, M. Sasaki, Phys. Rev. D 62, 024012 (2000). arXiv:gr-qc/9910076

9. S. Nojiri, O. Obregon, S.D. Odintsov, Phys. Rev. D 62, 104003 (2000). arXiv:hep-th/0005127

10. J. Ovalle, Int. J. Mod. Phys. D 18, 837 (2009). arXiv:0809.3547

11. J. Ovalle, Mod. Phys. Lett. A 23, 3247 (2008). arXiv:gr-qc/0703095
12. R. Casadio, J. Ovalle, Gen. Rel. Grav. 46, 1669 (2014)

13. J. Ovalle, F. Linares, Phys. Rev. D 88, 104026 (2013). arXiv: 1311.1844

14. J. Ovalle, L. Gergely, R. Casadio, Class. Quant. Grav. 32, 045015 (2015). arXiv:1405.0252

15. J. Ovalle, Int. J. Mod. Phys. Conf. Ser. 41, 1660132 (2016). arXiv: 1510.00855

16. R. Casadio, J. Ovalle, R. da Rocha, Class. Quant. Grav. 31, 045016 (2014). arXiv: 1310.5853

17. R. Casadio, J. Ovalle, R. da Rocha, Europhys. Lett. 110, 40003 (2015). arXiv:1503.02316

18. R.T. Cavalcanti, A. Goncalves da Silva, R. da Rocha, Class. Quant. Grav. 33, 215007 (2016). arXiv:1605.01271

19. S. Okuzumi, M. Sakagami, Phys. Rev. D 76, 084027 (2007). arXiv:gr-qc/0703070

20. E. Abdalla, R.A. Konoplya, A. Zhidenko, Class. Quant. Grav. 24, 5901-5910 (2007). arXiv:0706.2489

21. M.A. Cuyubamba, Class. Quant. Grav. 30, 195005 (2013). arXiv: 1304.3495

22. H. Furuhashi, Y. Nambu, H. Saida, Class. Quant. Grav. 23, $5417-$ 5438 (2006). arXiv:gr-qc/0601066

23. K.D. Kokkotas, B.G. Schmidt, Living Rev. Rel. 2, 2 (1999). arXiv:gr-qc/9909058

24. H.P. Nollert, Class. Quant. Grav. 16, R159-R216 (1999)

25. R.A. Konoplya, A. Zhidenko, Rev. Mod. Phys. 83, 793-836 (2011). arXiv: 1102.4014

26. C. Chirenti, A. Saa, J. Skakala, Phys. Rev. D 87, 044034 (2013). arXiv: 1211.1046

27. J. Morgan, V. Cardoso, A.S. Miranda, C. Molina, V.T. Zanchin, Phys. Rev. D 80, 024024 (2009). arXiv:0906.0064

28. C.H. Coimbra-Araujo, R. da Rocha, I.T. Pedron, Int. J. Mod. Phys. D 14, 1883-1898 (2005). arXiv:astro-ph/0505132

29. X.H. Ge, S.W. Kim, Phys. Lett. B 652, 349-358 (2007). arXiv:0705.1404

30. L. Randall, R. Sundrum, Phys. Rev. Lett. 83, 3370 (1999). arXiv:hep-ph/9905221

31. G.R. Dvali, G. Gabadadze, M. Porrati, Phys. Lett. B 485, 208-214 (2000). arXiv:hep-th/0005016

32. R. Casadio, R. da Rocha, Phys. Lett. B 763, 434 (2016). arXiv: 1610.01572

33. M. Gleiser, N. Jiang, Phys. Rev. D 92, 044046 (2015). arXiv: 1506.05722

34. K.C. Wong, K.S. Cheng, T. Harko, Eur. Phys. J. C 68, 241-253 (2010). arXiv:1005.3101

35. D. Bazeia, J.M. Hoff da Silva, R. da Rocha, Phys. Rev. D 90, 047902 (2014). arXiv:1401.6985

36. R. Da Rocha, A. Piloyan, A.M. Kuerten, C.H. Coimbra-Araujo, Class. Quant. Grav. 30, 045014 (2013). arXiv:1301.4483

37. L.D. Landau, E.M. Lifshitz, Fluid Mechanics, vol. 6, 2nd ed. in Course of Theoretical Physics (Pergamon Press, New York, 1987)

38. W.G. Unruh, Phys. Rev. D 51, 2827 (1995)

39. M. Visser, Class. Quant. Grav. 15, 1767 (1998). arXiv:gr-qc/9712010

40. C. Barcelo, S. Liberati, M. Visser, Living Rev. Rel. 8, 12 (2005). arXiv:gr-qc/0505065

41. L.C.B. Crispino, E.S. Oliveira, G.E.A. Matsas, Phys. Rev. D 76, 107502 (2007)

42. R. Schutzhold, W.G. Unruh, Phys. Rev. D 66, 044019 (2002). arXiv:gr-qc/0205099

43. R.A. Mosna, J.P.M. Pitelli, M. Richartz, Phys. Rev. D 94, 104065 (2016). arXiv:1611.09290

44. K. Lin, J. de Oliveira, E. Abdalla, Phys. Rev. D 90, 124071 (2014). arXiv: 1409.4066

45. R. Casadio, J. Ovalle, R. da Rocha, Class. Quant. Grav. 32, 215020 (2015). arXiv: 1503.02873 